define it. However, we need to investigate their real perceptions about this philosophy of care.

Aims: The aim of this research paper is to analyze how nurses from Gaslini Hospital perceive FCC practices. We use data taken from a questionnaire and compare these results with those obtained from other studies carried out in different countries.

Method: Qualitative study of data analysis from CYPAPT questionnaire.

Results: There is worldwide agreement about the features of FCC. Such agreement holds both for the positive sides, such as family individuality and trust in involving parents, and in weak points, such as structural and organizational problems and education paths for health professionals.

Conclusions: FCC is well rated worldwide from a theoretical point of view, but it needs further studies and multidisciplinary experimentations to be implemented in practice in the best way.

\title{
P008 - Perceptions of children and their parents about the pain experienced during their hospitalization and its impact on parents' quality of life
}

\author{
Vasiliki Matziou (Greece) ${ }^{1}$; Pantelis Perdikaris (Greece) ${ }^{2}$; \\ Efrosyni Vlahioti (Greece) ${ }^{3}$; Vasia Dimitriou (Greece) ${ }^{1}$; \\ Konstantinos Tsoumakas (Greece) ${ }^{1}$
}

${ }^{1}$ Faculty of Nursing, National \& Kapodestrian University of Athens; ${ }^{2}$ General Children's Hospital of Athens 'P \& A Kyriakou'; ${ }^{3}$ General Children's Hospital of Athens 'Agia Sophia'

Theme: Pain

Keywords: Adolescent, children, parents, perceptions, quality of life.

Background: In children and adolescents with cancer, as well as in their parents, the quality of life is affected due to pain experienced during their hospitalization.

Objectives: The aim was to report and assess paediatric and parental opinions about the pain the paediatric patients suffer.

Methods: The sample of the study consisted of 92 paediatric cancer patients, 159 paediatric patients with musculoskeletal problems and one of their parents with the Paediatric Pain Questionnaire and the PedsQL-Family Impact Module.

Results: Young patients reported higher acute pain scores than their parents $(z=-2.5$, $p=0.011 ; 99 \% \mathrm{Cl}: 0.008-0.013)$. Young patients with orthopaedic disorders had higher acute and chronic pain scores in comparison to their parents' reports $(z=-3.4, p=0.001$; 99\% Cl: $0.000-0.001$ and $z=-2.3, p=0.021 ; 99 \% \mathrm{Cl}: 0.017-0.025$ respectively).

Conclusions: The parental quality of life is affected especially when the pain is caused by life-threatening diseases, but it improves as the treatment ends. 